\title{
IMPLEMENTING PACKET BROADCASTING AlgorithM OF MIMO BASEd MOBILE AD-HOC NETWORKS TO EXPLOIT DIVERSITY
}

\author{
Swati Chowdhuri ${ }^{1}$, Santanu Maiti ${ }^{2}$, Dr. Sheli Sinha Chaudhuri ${ }^{3}$ and Dr. \\ P.K.Baneerjee ${ }^{4}$ \\ ${ }^{1,2}$ Asst.Professor, ECE, Seacom Engineering College, Howrah-711302 \\ ${ }^{3}$ Associate Professor, ETCE, Jadavpur University, kolkata-700032 \\ ${ }^{4}$ Professor, ETCE, Jadavpur University, kolkata-700032
}

\begin{abstract}
With the rapid growth of wireless communication infras, tructure over the recent few years, new challenges has been posed on the system and analysis on wireless adhoc networking. Implementation of MIMO communication in such type of network is enhancing the packet transmission capabilities. There are different techniques for cooperative transmission and broadcasting packet in MIMO equipped Mobile Adhoc Network. We have employed a model network in the OPNET environment and propose a new scheduling algorithm based on investigating the different broadcasting algorithm. The new broadcasting algorithm improves the packet transmission rate of the network based on energy performance of the network and minimizes the BER for different transmission mode which is illustrated in this paper. The simulations are done in MATLAB and OPNET environment and the simulated result for the packet transmission rate are collected and shown in the tabular form. Also simulate the network for generating a comparative statement for each mobile node. And performance analysis is also done for the model network. The main focus is to minimize BER and improve information efficiency of the network.
\end{abstract}

\section{KEYWORDS}

Multi Input Multi Output (MIMO), adhoc network, cooperative transmission, opportunistic large array, packet broadcast.

\section{INTRODUCTION}

The major elements for successful implementation of MIMO communication systems are coding and signal processing. The signal transmission technique using multiple antennas at the two sides of a communication links has a great potential to significantly improve the transmission efficiency of the wireless communication system without the need for extra operational frequency bandwidth $[1,2,3]$. To improve the spectral efficiency, MIMO antenna elements are integrated to the transmitting and receiving links of the communication systems. Multiple antenna elements with associated signal processing algorithm have to be implemented at mobile nodes. A new scheduling algorithm is discussed which is related with the firing of the network nodes. These changes the energy of the network i.e. transmission efficiency of the network by discussing Energy-Performance Metric (EPM). Packet transmission is an important parameter of the considering network which is depends on minimization of BER and improve packet transmission rate. In this chapter we have implemented the packet broadcasting algorithm of MIMO based Mobile adhoc network for exploiting diversity. In Mobile adhoc

DOI : $10.5121 /$ ijnsa.2014.6203 
network, conventional flooding broadcast mechanism has some limitation. We have implemented a physical layer technique that is cooperative transmission which is a much more efficient method than traditional flooding broadcast [3,4]. This new technique allows transmission collectively i.e. not to be capable to forward the data individually from a set of asynchronous transmitter to a remote destination. The multiple transmitter acts as an antenna array-an Opportunistic Large Array, or OLA - to transmit the message from set of asynchronous transmitter to remote destination. The transmission algorithm is designed in such a manner to exploit the diversity and improve the Network Utility Function (NUF).

\section{CoOperative Transmission Mechanism}

Cooperative transmission (CT) mechanism is based on resource sharing method. Here two or more node cooperates to transmit the same message as a result two or more single antenna transmitters achieve the benefits of an array transmitter $[5,6]$. The key idea in user-cooperation is that of resource-sharing among multiple nodes in a network. The main motive of exploration of user-cooperation is that to share power and computation with neighboring nodes can lead to save the overall network resources. Conventional flooding mechanism has some limitation due to installation cost of multiple antennas on the terminals, additional processing time and the coherence distance between the transmitting and receiving antennas. To minimize processing time and coherence distance long range communication is simply impossible. A single long range link with a chain is replace by a number of short range links by placing a series of nodes in between the source and destination [7,8]. In the Mobile adhoc network, cooperative transmission is about exploiting the broadcasting nature by transforming single-antenna terminals into a virtual antenna array. Thus, the multiple signals are transmitted from source to destination terminals through different uncorrelated channels using Relay terminal provide benefits of spatial diversity. An example of single-relay cooperative scenarios is shown in Figure 1. Source terminal (S) transmits a signal to the destination terminal (D) through two paths for overhear the signal. One is direct path (S-D) and other one is via the relay terminal R. When Relay terminal $(\mathrm{R})$ is in a cooperative mode, it forwards the source message to the destination $\mathrm{D}$. As a result $\mathrm{D}$ receives two signals: the original one transmitted from $\mathrm{S}$ through the direct path (S-D) and the relayed one forwarded by R through the relayed path (S-R-D). These two received signals are combined at the destination terminal to achieve a better spatial diversity compared to the receive signal achieved with a single direct path.

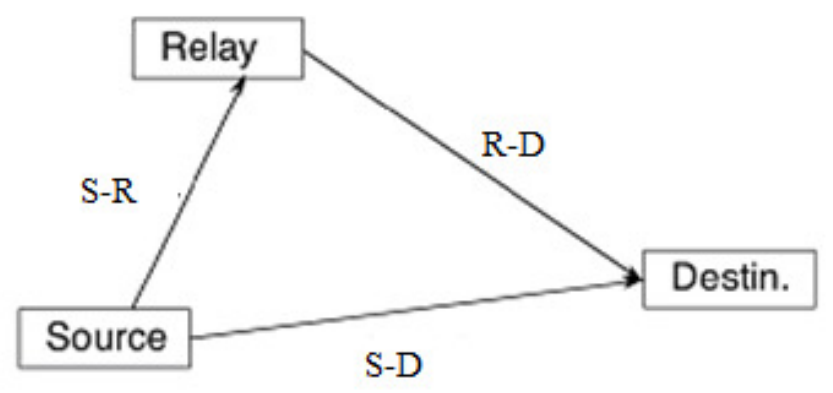

Figure 1: Example of Cooperative Transmission

The main cooperation is done by intermediate nodes of the network along the data transmission route for the multi-hop networks. A single relay terminal or a set of relay terminals helps on data relaying from a previous terminal (source terminal) to a next-hop terminal (destination terminal) $[9,10]$. Spatial diversity is the main advantage provided by cooperative 
communications. This property can be expressed in terms of increased diversity order. The Amplify-and-Forward (AF) scheme is a cooperative transmission scheme.

\section{Algorithms For Cooperative Transmission}

To exploit the multiuser diversity and traffic demand for channel condition an integrated scheduling scheme is proposed here. This scheduling algorithm takes the advantages of the random topology of MIMO based adhoc networks. A group of nodes forming a mesh network take the advantages of cooperative transmission; the sender node can transmit multiple data to downstream nodes or receiving nodes, while a receiver can receive packets from upstream node or transmitting nodes. The data scheduling schemes used here operate in cross layers and with the consideration of physical channel condition and service requirement of the user traffic. The scheduling requests can be categorized as centralized scheduling, hierarchical scheduling and decentralized scheduling based on their system architecture. In centralized scheduling, central scheduler collects the information of network resources. In Hierarchical system different scheduling policy is used for local and global scheduling, and this does not depends on the single scheduler. In decentralized scheduling the distributed schedulers interact with each other and schedule jobs at remote nodes for execution. Local schedulers can submit jobs to the receiver or receive from transmitter through direct communication or a central job pool. In the simulation environment we use centralized scheduling algorithm.

In this algorithm the scheduling time depends on the processing time of the nodes of the network and firing time of the node. Data to network bandwidth consists of information about the requirement on the resource that is necessary to execute a particular job firing for the node in the network environment. The congestion control process decides which packets to be injected for transmission. The next packet to be transmitted is determined by the job-scheduling algorithm. Mobile adhoc scheduling algorithms are responsible for firing of the network nodes as well as the job scheduling process. Scheduling requests for firing node can be controlled by Network Utility Maximization (NUM) which is defined as

$$
\begin{aligned}
& \operatorname{Max} \sum U_{f}\left(X_{f}\right) \\
& \text { subject to } \\
& \sum\left(X_{f}\right) \leq C_{e} \text { where } f \in S_{e}
\end{aligned}
$$

where $C_{e}$ be the capacity of the server, $S_{e}$ be the set of flows passing through the server and $X_{f}$ be the current injection rate into flow f. $U_{f}($.$) is defined as the utility function. Network$ utility function represents the network efficiency by the given packet transmission rate and Energy Performance Metric (EPM) [16]. Joint Energy Performance Metric (EPM) for mobile adhoc networks explain the characteristics of the routing protocols for both performance and energy consumption. A good metric will capture the average behavior of a system, as commoncase events determine the total utilization of a network. A family of energy-performance metrics is defined in our proposed scheduling method using the following equations:

\section{$\operatorname{EPM}(\alpha)=($ Averageenergy $)(\text { Averageperformance })^{-\alpha}$}

where $\alpha$ is a parameter that determines the weighting between energy and performance $[11,12]$. We first measure the energy and performance metric of the MIMO based Mobile adhoc network to evaluate the algorithm. The average energy of a network is simply the total network energy over the number of nodes. Measuring average network performance of an adhoc network is more difficult. We choose to measure the average performance of a network based 
on its ability to successfully deliver packets. The transmission efficiency is defined as the ratio of Network packet received to Network packet transmitted. Now substituting these definitions into the prior definition of $\operatorname{EPM}(\alpha)$ we get,

$\operatorname{EPM}(\alpha)=\left(\right.$ Network energy per node) $(\text { Transmission efficiency })^{-\alpha}$

$\operatorname{EPM}(\alpha)=($ Network energy/ node $)(\text { Network packet transmitted/network packet received })^{-\alpha}$

The choice of $\alpha$ is more important and difficult to get suitable EPM metric (the unit of energy). At $E P M(0)$ the metric reduces to a pure energy metric and for $E P M(\infty)$ the metric is a pure performance metric. The main goal is to use a metric with a value somewhere in between these extremes. We will initially assume the value of $\alpha$ to be one and show empirically that this is a reasonable metric parameter for adhoc network routing protocols.

\section{Cooperative Transmission is summarized as follows:}

- Downstream transmission: Sender node transmit Upstream Transmission: Receiver node receive

- Considering Scheduling algorithm as centralized, based on physical channel condition of the network and network traffic

- Node firing is related with the processing time which is determined by scheduling algorithm for Network Utility Maximization (NUM)

- Network Utility Function (NUF) depends on Packet transmission rate and Energy Performance Metric (EPM).

- EPM depends on parameter $\alpha$, $\alpha=0$, Pure energy metric $\alpha=1$, Pure performance metric.

\section{Simulation Model AND ReSults}

We have a randomly deployed adhoc network with 40 number of nodes in the area of 10,000 $(100 * 100)$ sqft in the OPNET environment. The model is devised to operate the nodes in AODV protocol based on IEEE $802.11 \mathrm{~b}$ distribution coordination function (DCF). The deployed network is shown in Figure 2.

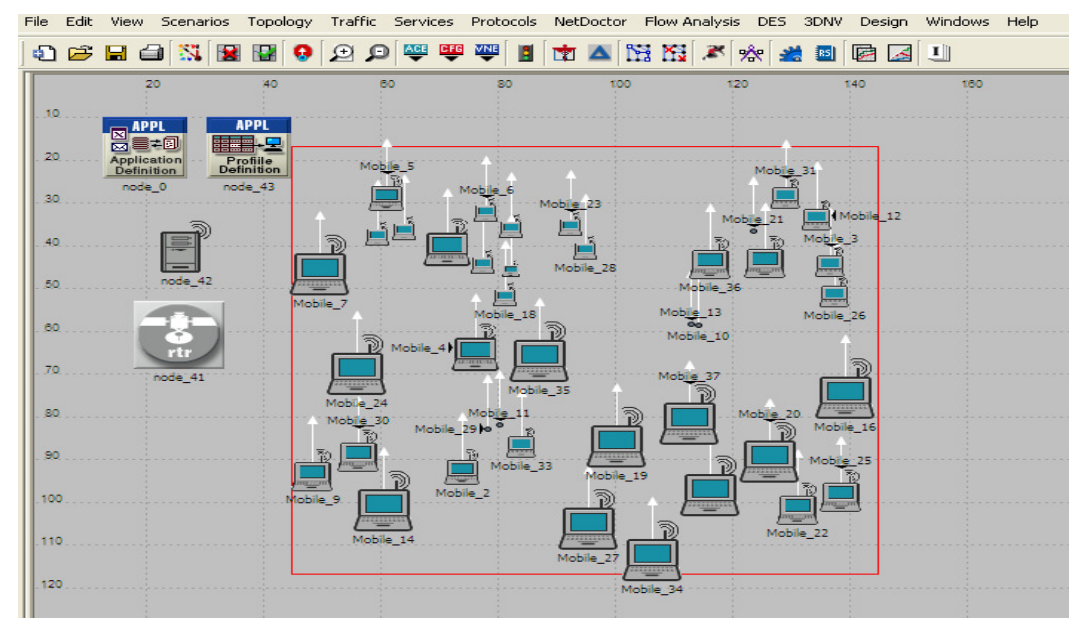

Figure 2: Randomly distributed MANET (deployed network) 
International Journal of Network Security \& Its Applications (IJNSA), Vol.6, No.2, March 2014

The selected network environment is shown in Table 1 .

Table 1:

\begin{tabular}{|l|l|}
\hline $\begin{array}{l}\text { Node Transmission } \\
\text { Power }\end{array}$ & $.005 \mathrm{Watt}$ \\
\hline Operational Mode & $802.11 \mathrm{~b}$ standard \\
\hline Data rate & $11 \mathrm{Mbps}$ \\
\hline Adhoc routing protocol & AODV \\
\hline Technology & WLAN (adhoc) \\
\hline
\end{tabular}

As the power consumption is a major factor of the adhoc network we have set the minimum power for the node. The operating power of each node of the network is set to $5 \mathrm{mWatt}$. We have seen that if the power set is less then there is more packet failure with the time, by considering the different power we have judiciously made the choice of the $5 \mathrm{mWatt}$ power.

\subsection{Minimization of Bit Error Rate (BER)}

The large-scale natures of the adhoc network consisting of a large number of nodes in dense deployments naturally encourage the use of distributed arrays of nodes. OLA based broadcast transmission minimizes Bit Error rate (BER) in different transmission mode (Half duplex, Full duplex) after considering Maximal Ratio Combining (MRC) non cooperative broadcast. BER at different transmission mode and BER on OLA performance of the network are shown in Figure $3 a$ and $3 b$.

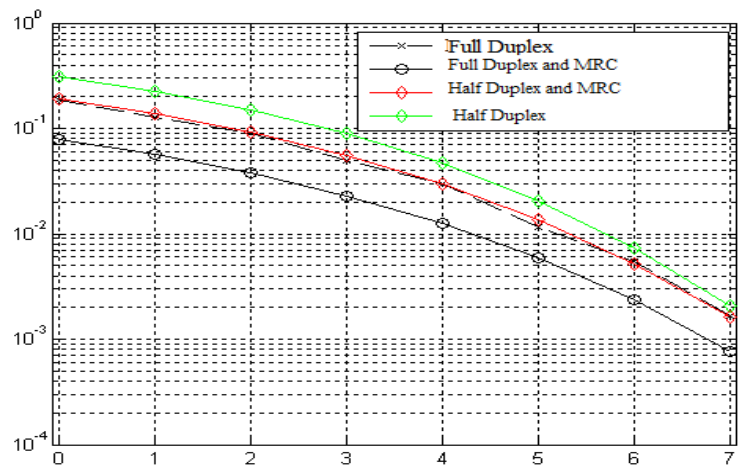

Figure 3a: BER vs SNR at different transmission mode 


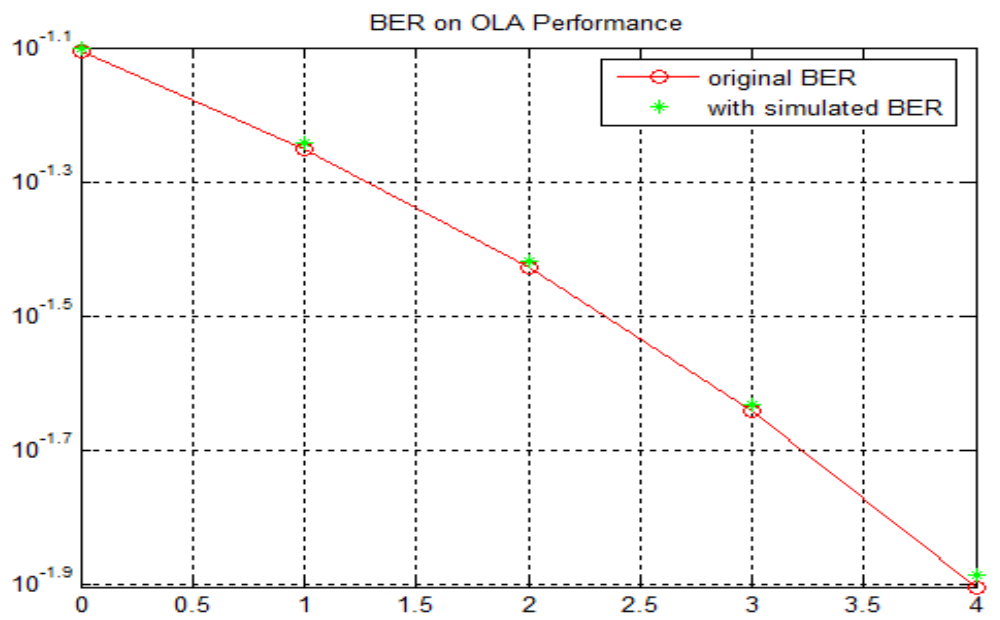

Figure 3b: BER on OLA performance

\subsection{Packet transmission analysis of the model network}

The deployed network in the OPNET environment is shown in Figure 2 is used to measure the packet transmission rate through simulation. The simulated result shown in Figure $4 \mathrm{a}, 4 \mathrm{~b}$ and $4 \mathrm{c}$ indicates the processing and transmission of packet with time. Packet transmission with time is shown in table 2. The performance of different node in terms of their packet created, copied and destroyed is shown in table 3. A comparative study is made for packet transmission of each 40 nodes of the network in Figure 5. The packet lost for MANET Gateway in the network is very small or zero. During simulation the supporting devices like application config (node_0) and profile config (node_43) don't take direct role regarding packet transmission in the network. After implementation of the algorithm the simulated results shows time for packet delivery process and firing of the node. As describe in the algorithm node firing is depends on the time which is determined the scheduling algorithm. Ethernet delay is calculated based on processing time of the packet transmission for the node consisting of the network. Firing of each adhoc node and Ethernet delay is shown in Figure 7 and 8.

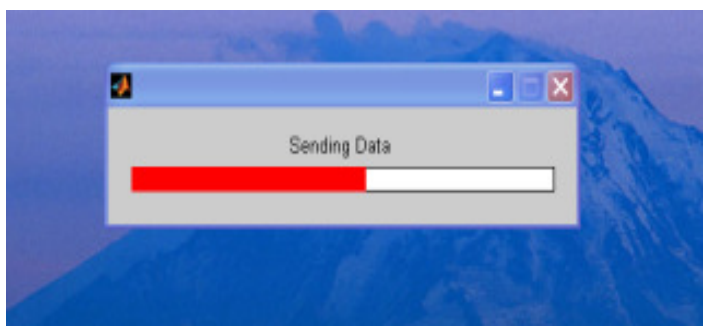

Figure 4a: 50\% Packet sent 
International Journal of Network Security \& Its Applications (IJNSA), Vol.6, No.2, March 2014

Figure 4b: $75 \%$ Packet sent
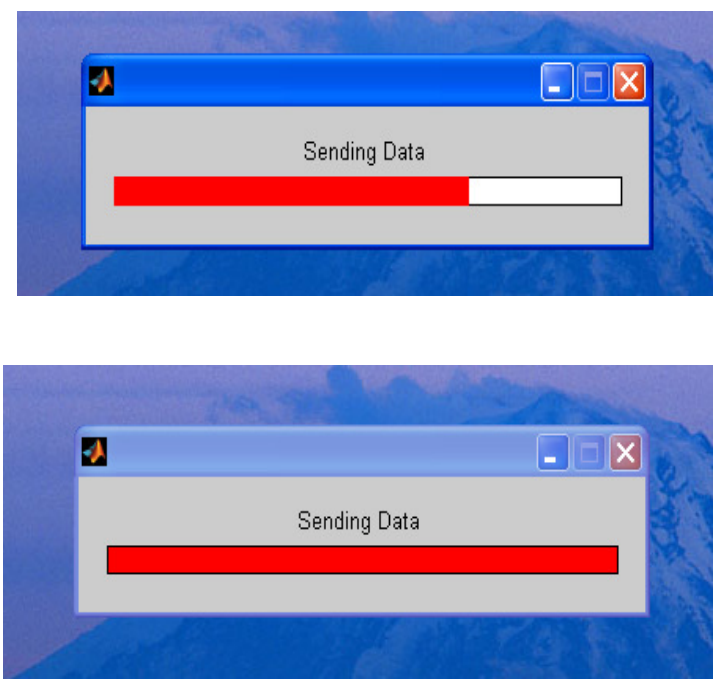

Figure 4c: 100\% Packet sent

Packet transmission rate is shown in Table 2.

Table 2:

\begin{tabular}{|l|l|}
\hline Packet transmission in percentage & Time in sec \\
\hline $50 \%$ & 45 \\
\hline $75 \%$ & 66 \\
\hline $100 \%$ & 92 \\
\hline
\end{tabular}


International Journal of Network Security \& Its Applications (IJNSA), Vol.6, No.2, March 2014

Packet transmission strategies for the model network are shown in Table 3.

Table 3:

\begin{tabular}{|c|c|c|c|}
\hline Node Name & Created & Copied & Destroyed \\
\hline node_o & 0 & 0 & 0 \\
\hline node_1 & 26 & 8 & 24 \\
\hline node_10 & 35 & 12 & 33 \\
\hline node_11 & 17 & 4 & 15 \\
\hline node_12 & 17 & 4 & 15 \\
\hline node_13 & 17 & 4 & 15 \\
\hline node_14 & 17 & 4 & 15 \\
\hline node_15 & 17 & 4 & 15 \\
\hline node_16 & 17 & 4 & 15 \\
\hline node_17 & 14 & 4 & 15 \\
\hline hode_18 & 17 & 4 & 15 \\
\hline node_19 & 26 & 8 & 24 \\
\hline node_2 & 17 & 4 & 15 \\
\hline node_20 & 17 & 4 & 15 \\
\hline hode_21 & 17 & 4 & 15 \\
\hline hode_22 & 17 & 4 & 15 \\
\hline node_23 & 17 & 4 & 15 \\
\hline node_24 & 17 & 4 & 15 \\
\hline hode_25 & 17 & 4 & 15 \\
\hline hode_26 & 17 & 4 & 15 \\
\hline node_27 & 26 & 8 & 21 \\
\hline node_28 & 17 & 4 & 15 \\
\hline node_29 & 26 & 8 & 24 \\
\hline node_3 & 17 & 4 & 15 \\
\hline hode_30 & 17 & 4 & 15 \\
\hline node_31 & 17 & 4 & 15 \\
\hline hode_32 & 17 & 4 & 15 \\
\hline hode_33 & 17 & 4 & 15 \\
\hline hode_34 & 17 & 4 & 15 \\
\hline hode_35 & 26 & 8 & 24 \\
\hline hode_36 & 17 & 4 & 15 \\
\hline hode_37 & 26 & 8 & 24 \\
\hline hode_38 & 17 & 4 & 15 \\
\hline hode_39 & 17 & 4 & 15 \\
\hline hode_4 & 17 & 4 & 15 \\
\hline hode_40 & 17 & 4 & 15 \\
\hline node_41 & 0 & 117 & 82 \\
\hline hode_42 & 813 & 240 & 360 \\
\hline hode_43 & 0 & 0 & 0 \\
\hline hode_5 & 17 & 4 & 15 \\
\hline hode_6 & 17 & 4 & 15 \\
\hline hode_7 & 17 & 4 & 15 \\
\hline hode_8 & 17 & 4 & 15 \\
\hline hode_9 & 17 & 4 & 15 \\
\hline [Total] & 1562 & 549 & 1111 \\
\hline
\end{tabular}


International Journal of Network Security \& Its Applications (IJNSA), Vol.6, No.2, March 2014

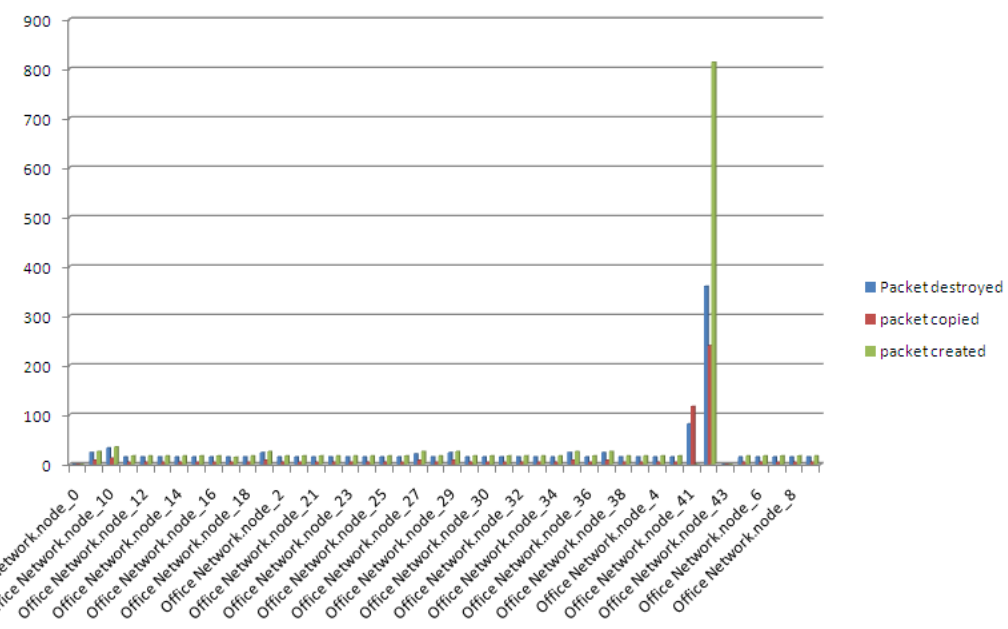

Figure 5: Comparative study of packet transmission strategies

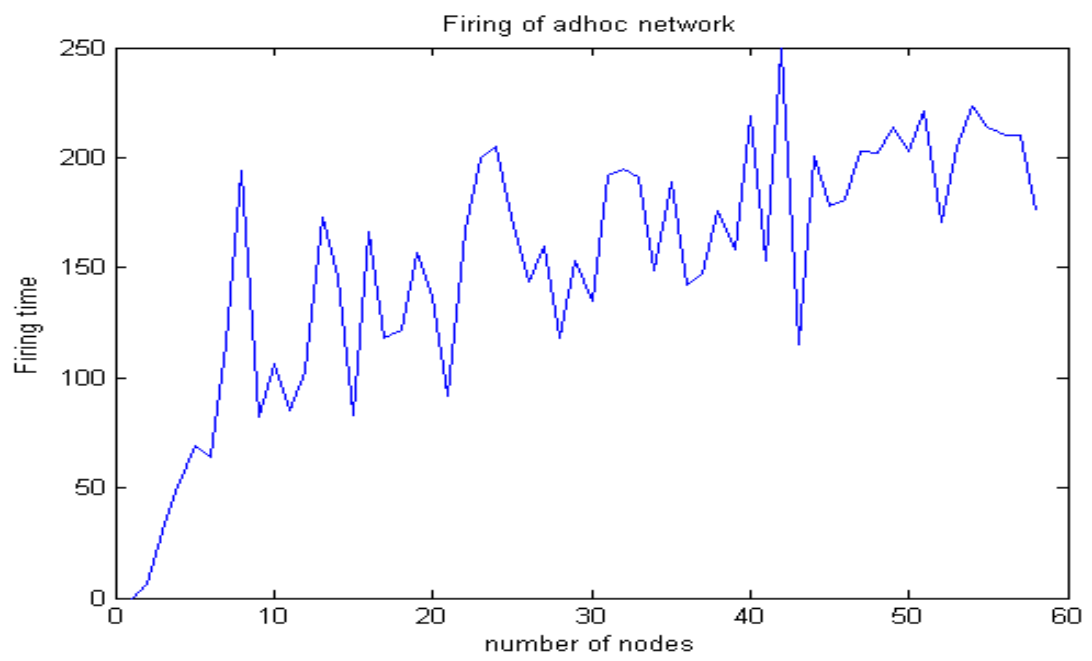

Figure 6:Firing of adhoc node

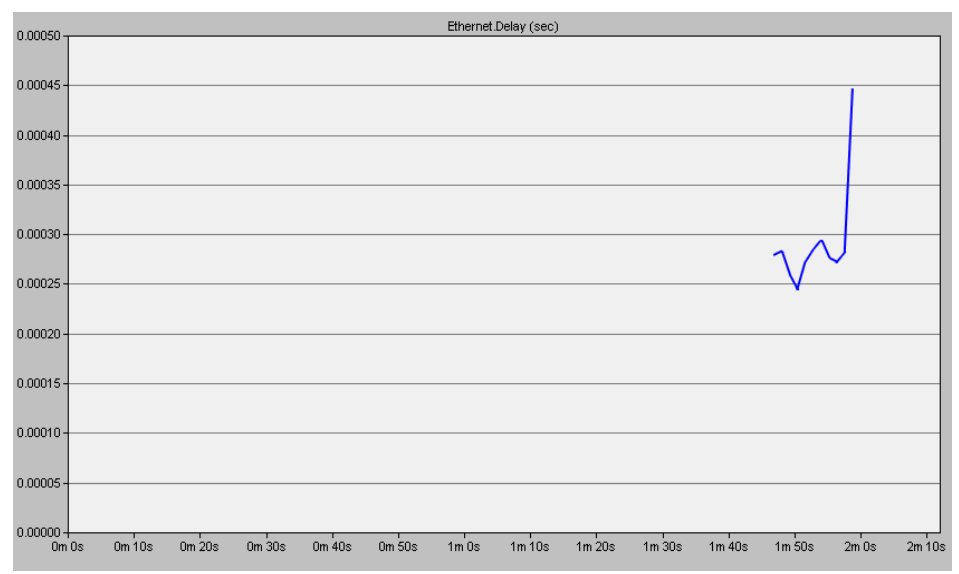

Figure 7: Ethernet Delay for each node in the model Network 


\section{CONCLUSIONS}

In this chapter we have implemented a broadcasting algorithm in the MIMO based Mobile adhoc network. We have analyzed and simulated the deployed network in the OPNET environment. The simulated result shows that the BER is minimized after considering MRC (Maximal Ratio Combining) in different transmission mode (Full duplex and Half duplex). The simulated result also shows a comparative study of packet transmission strategy (Packet created, Copied and destroyed) of each node of the network. The processing time of the network which is related with packet delivery process and firing of the node is also evaluated. The packet transmission rate of the network is increased after implementing the algorithm. This type of algorithm not only uses to minimize BER but also increases the packet transmission rate. In the hostile environment where BER is too high, this algorithm is more advantageous to minimize the BER and increase the packet transmission rate.

\section{REFERENCES}

[1] Anna Scaglione,, "Opportunistic Large Arrays: Cooperative Transmission in Wireless Multihop Adhoc Networks to Reach Far Distances," IEEE Transaction on Signal Processing, Vol.51, No.8, Aug 2003.

[2] Vandana Rohokale, Nandkumar Kulkarni, Horia Cornean, "Cooperative Opportunistic Large Array Approach for Cognitive Radio Networks" 978-1-4244-6363-3/10/\$26.00c 2010 IEEE.

[3] Edoardo S. Biagioni "Algorithms for Communication in Wireless multi-hop adhoc Networks using Broadcasts in Opportunistic Large Arrays (OLA)".

[4] Lakshmi Thanayankizil and Mary Ann Ingram, "Reactive Routing For Multi-hop Dynamic Adhoc Networks Based On Opportunistic Large Arrays," in Proc. IEEE Military Commun. Conf. (MILCOM), Oct. 2000, vol. 2, pp. 861-865.

[5]. L. Thanayankizil, A. Kailas, and M. A. Ingram, "A simple cooperative transmission protocol for Energy efficient broadcasting over multi-hop wireless networks", KICS/IEEE Journal of Communications and Networks, vol.10, no.2, pp.213-220, June 2008.

[6]. Lakshmi V. Thanayankizil, Aravind Kailas, Mary Ann Ingram, "Energy- Efficient Strategies for Cooperative Communications in Wireless Sensor Networks," sensor comm, pp.541-546, 2007 International Conference on Sensor Technologies and Applications (SENSORCOMM 2007), 2007.

[7] Thesis on Cooperative Communications in Adhoc Networks by Wasimon Panichpattanakul.

[8] B.Chen and M.J. Gans, "MIMO communications in adhoc networks." IEEE transactions on signal processing, vol 54, no 7, July 2006.

[9] Pei Liu, Zhifeng Tao, Zinan Lin, Elza Erkip, and Shivendra Panwa," Cooperative Wireless Communications: A Cross-Layer Approach” IEEE Wireless Communications August 2006.

[10] Edoardo S. Biagioni "Collision Free Broadcasting in Wireless AdHoc Networks using Cooperativ Diversity".

[11] A. Nosratinia, T. E. Hunter and A. Hedayat, "Cooperative communication in wireless networks," IEEE Communications Magazine, vol. 42, pp. 74-80, Oct. 2004.

[12] S.M. Alamouti. A simple transmit diversity technique for wireless communications. IEEE JSAC, 16(8):14511458, 1998.

\section{Authors:}

Swati Chowdhuri: Presently, she is an Asst.Professor of Seacom Engineering College. She has been completed B.E. in Electronics and communication Engg from Burdwan University with Honours in the year of 2005 and completed M.Tech in Computer Technology from Jadavpur University in the year of 2009. Her research interests in the field of "MIMO based Mobile Ad-hoc networks".

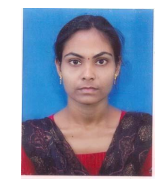

